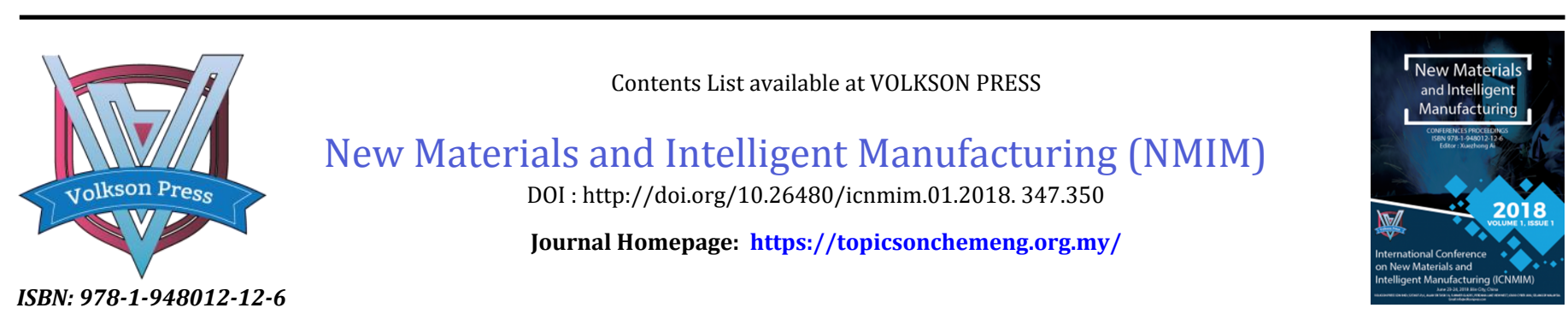

SBN: 978-1-948012-12-6

\title{
A NEW INTEGER-VALUED AUTO-REGRESSIVE MODEL BASED ON BINOMIAL THINNING OPERATOR
}

\author{
Qingchun Zhang*, Xiaodong Fan \\ School of science, Jilin Institute of Chemical Technology, 45 Chengde Street, Jilin City, China \\ *Corresponding Author Email: zqingchun@126.com
}

This is an open access article distributed under the Creative Commons Attribution License, which permits unrestricted use, distribution, and reproduction in any medium, provided the original work is properly cited.

\section{ARTICLE DETAILS}

Article History:

Received 26 June 2018 Accepted 2 July 2018

Available online 1 August 2018

\begin{abstract}
The integer-valued time series appears in many areas of real life, for example, medical care, economics, transportation, insurance, finance, and so on. Especially, we are in the big data period and all kinds of dispersed integer-valued data may appear everyday. The thinning operator is the main method to study the integer-valued time series. In this paper, we propose a new stationary integer-valued first-order auto-regressive (INAR(1)) model based on binomial thinning operator. The model is constructed by considering the innovation to fit different dispersed data. The definition and statistical properties of the model are given. The maximum likelihood method is applied to estimate the parameters. We considered one specific model, in which the innovation follows geometric distribution, which can deal with over-dispersed integer-valued time series data. The performance of CML estimators are evaluated by simulation study. An application is given to an offence data in Track 1706 in Pittsburgh.
\end{abstract}

\section{KEYWORDS}

Binomial thinning operator, Over-dispersed, Parameter estimation, Geometric distribution.

\section{INTRODUCTION}

The integer-valued time series appears in many areas of real life, for example, medical care, economics, transportation, insurance, finance, and so on. Especially, we are in the big data period and all kinds of dispersed integer-valued data may appear everyday $[1,2]$. So, it is important and necessary to construct the appropriate model to fit the data. The integer auto- regressive (INAR) model is a typical example of the thinning model to fit the integer-valued data. There are researcher introduced binomial thinning operator, which drives the development of the integer-valued time series [3]. In 1987, scientist has given the definition of the INAR(1) model based on binomial thinning operator [4]. They considered the Poisson distribution as the innovation of model to establish the INAR(1) model based on binomial thinning operator. A studied the estimates methods of INAR(1) model [5,6]. In a studied the properties of the counting time series based on binomial thinning [7]. Another researchers also studied the compound Poisson INAR(1) model, cumulative functions, and estimates of the INAR(1) with binomial thinning operator and got good results [8].

In China, there are not many research results on the integer autoregressive (INAR) model based on binomial thinning operator. Some researchers proposed the INARGARCH model to study the zero inflated data [9]. There are researchers also established INAR(1) models based binomial thinning for a class of renew sequences and gave the statistical inferences of the model [10]. They has considered an INAR(1) model with binomial thinning for missing data and obtained some properties of the model [11]. At present, few people have studied the case of all kinds of dispersed data by considering innovation of the INAR(1) model based on binomial thinning operator. This is exactly what this paper is going to study.

\section{BINOMIAL THINNING OPERATOR AND OVERDISPERSED}

Definition 2.1 Binomial thinning operator (Steual and van Harn (1979)) is defined as follows

$$
\alpha \circ X=\sum_{i=1}^{X} Y_{i}
$$

where $\left\{Y_{i}\right\}_{i=1}^{X}$ is a sequence of independent and identical distributions (i.i.d.) of random variables following the Bernoulli distribution with parameters, that is, $Y_{i} \sim B(1, \alpha)$ and its distribution law is following

$$
P\left(Y_{i}=1\right)=\alpha=1-P\left(Y_{i}=0\right), \alpha \in[0,1] \text {. }
$$

Definition 2.2 Assume that the expectation and variance of the stationary process $\left\{X_{t}\right\}$ be $E\left(X_{t}\right)=\mu, \operatorname{Var}\left(X_{t}\right)=\sigma^{2}$. Let $d=\frac{\sigma^{2}}{\mu}$, then $\left\{X_{t}\right\}$ is over-dispersed if $d>1[8]$.

\section{INAR (1) MODEL BASED ON BINOMIAL THINNING}

\subsection{Model}

Definition 3.1 The following process $\left\{X_{t}\right\}$ is said to be a first-order integer-valued auto-regressive process based on binomial thinning operator, simply denoted as the BINAR (1) model [4].

$X_{t}=\alpha \circ X_{t-1}+\varepsilon_{t}, \quad t \in Z$,

Where" $\circ$ "is a binomial thinning operator defined by Definition 2.1, and $\left\{\varepsilon_{t}\right\}$ is a sequence of i.i.d random variables. Assume that it is independent of within thinning operators and of $\left\{\varepsilon_{t}\right\}$,and for $\forall s<t$, $\operatorname{cov}\left(X_{s}, \varepsilon_{t}\right)=0$. Furthermore, we assume that $E\left(\varepsilon_{t}\right)=\lambda$, $\operatorname{Var}\left(\varepsilon_{t}\right)=V \lambda, V>0$.

Some statistical properties of the BINAR(1) process $\left\{X_{t}\right\}$ are given as follows. 


\section{Proposition 3.1}

$$
\begin{aligned}
& \text { (1) } E\left(X_{t}\right)=\frac{\lambda}{1-\alpha} ; \\
& \text { (2) } \operatorname{Var}\left(X_{t}\right)=\frac{(\alpha+V) \lambda}{1-\alpha^{2}} ; \\
& \text { (3) } \operatorname{Cov}\left(X_{t}, X_{t+h}\right)=\alpha^{h} \operatorname{Var}\left(X_{t}\right), h=1,2, \cdots ; \\
& \text { (4) } E\left(X_{t} \mid X_{t-1}\right)=\alpha X_{t-1}+\lambda ; \\
& \text { (5) } \operatorname{Var}\left(X_{t} \mid X_{t-1}\right)=\alpha(1-\alpha) X_{t-1}+V \lambda ; \\
& \text { (6) } \operatorname{Cov}\left(X_{t} \mid X_{t-1}\right)=\alpha\left[\alpha(1-\alpha) X_{t-1}+\lambda V\right] .
\end{aligned}
$$

\subsection{Parameter Estimation}

The conditional maximum likelihood method is applied to estimate the unknown parameters in the BINAR (1) model. The conditional probability mass function of the BINAR(1) model can be expressed in the following form

$$
p\left(x_{t} \mid x_{t-1}, \theta\right)=\sum_{k=0}^{m} p_{1}\left(x_{t}-k\right) p_{2}(k),
$$

where

$p_{1}\left(x_{t}-k\right)=\left(\begin{array}{c}x_{t-1} \\ x_{t}-k\end{array}\right) \alpha^{x_{t}-k}(1-\alpha)^{x_{t-1}-x_{t}+k}$,

and $p_{2}(k)$ is the probability distribution of the innovation

$P\left\{\varepsilon_{t}=k\right\}, m=\min \left(x_{t}, x_{t-1}\right), \theta$ are unknown parameters.

Thus, the conditional likelihood function can be given as follows

$$
L(\theta \mid x)=\prod_{t=1}^{T} p\left(x_{t} \mid x_{t-1}, \theta\right)
$$

where $T$ is the sample size.

We gave a specific model by considering geometric distribution as the innovation of the BINAR(1) model in next section.

\section{BINAR (1) MODEL WITH GEOMETRIC INNOVATION}

\subsection{Model}

Definition 4.1 Assume that the innovation in BINAR(1) model defined in definition 3.1 follows a geometric distribution with parameter $\lambda$, that is, $\varepsilon_{t} \sim \operatorname{geo}\left(\frac{\lambda}{1+\lambda}\right)$,

and its distribution law is as follows
$P\left\{\varepsilon_{t}=k\right\}=\frac{\lambda^{k}}{(1+\lambda)^{k+1}}, \lambda>0, k=0,1,2, \cdots .(4.1)$

Then the model is called BINAR (1) model with geometric innovation denoted as GBINAR (1) model.

It is easy to see that the geometric distribution is over-dispersed as $E\left(\varepsilon_{t}\right)=\lambda, \operatorname{Var}\left(\varepsilon_{t}\right)=\lambda(1+\lambda)$, thus $V=1+\lambda$ in definition 3.1. By substituting $V=1+\lambda$ into proposition 3.1, the statistical properties of the BINAR (1) model with geometric innovation can be obtained.

Note that BINAR (1) model with geometric innovation is over-dispersed, which can be seen from

$d=\frac{\operatorname{Var}\left(X_{t}\right)}{E\left(X_{t}\right)}=1+\frac{\lambda}{1+\alpha}>1$

\subsection{Conditional Maximum Likelihood Estimate}

By substituting (4.1) into (3.2), the conditional probability distribution of the BINAR (1) model with innovation of geometric distribution can be obtained as follows

$$
p\left(x_{t} \mid x_{t-1}, \alpha, \lambda\right)=\sum_{k=0}^{m}\left(\begin{array}{c}
x_{t-1} \\
x_{t}-k
\end{array}\right) \alpha^{x_{t}-k}(1-\alpha)^{x_{t-1}-x_{t}+k} \frac{\lambda^{k}}{(1+\lambda)^{1+k}}, k=0,1,2, \cdots .
$$

where $m=\min \left(x_{t}, x_{t-1}\right)$, furthermore, we can get the conditional likelihood function of the BINAR(1) model with geometric innovation in the following

$$
L(\alpha, \lambda \mid x)=\prod_{t=1}^{T} p\left(x_{t} \mid x_{t-1}, \alpha, \lambda\right)
$$

where $T$ is the sample size.

\section{NUMERICAL SIMULATION}

We selected three sets of different parameter values for the INAR (1) process with geometric innovation. Then software $\mathrm{R}$ is used to generate random numbers from this model, and unknown parameters $\alpha$ and $\lambda$ are estimated by CML method using the random numbers generated. The results are summarized in Table 1 . We calculated the mean, empirical deviation(bias), and mean square error(sd) of each estimate, which are denoted as mean (bias, sd) in the table,for example, 0.1090 ( $0.0090,0.1144)$ represents that the mean of the estimate is 0.1090 , the estimated deviation is 0.0090 and a standard deviation is 0.1144 . All the simulations are based on 1000 replications, where the sample size are $\mathrm{n}=$ 50,100 , and 300 , respectively.

Table 1: CML Estimation for GBINAR(1) Model

\begin{tabular}{|ccc|}
\hline $\begin{array}{c}\text { True values } \\
\text { Sample sizes }\end{array}$ & $\hat{\alpha}^{C M L}$ & \\
$\alpha=0.1, \lambda=0.3$ & ias,sd) & \\
$\mathrm{n}=50$ & & $0.2915(-0.0085,0.0924)$ \\
$\mathrm{n}=100$ & $0.1090(0.0090,0.1144)$ & $0.2975(-0.0025,0.0669)$ \\
$\mathrm{n}=300$ & $0.1031(0.0031,0.0863)$ & $0.2975(-0.0025,0.0669)$ \\
$\alpha=0.3, \lambda=1$ & $0.0999(-1 \mathrm{e}-04,0.0551)$ & \\
$\mathrm{n}=50$ & $0.2890(-0.0111,0.1004)$ & $1.0085(0.0085,0.2304)$ \\
$\mathrm{n}=100$ & $0.2963(-0.0037,0.0707)$ & $1.0077(0.0077,0.1638)$ \\
$\mathrm{n}=300$ & $0.2994(-0.0006,0.0403)$ & $1.0013(0.0013,0.0962)$ \\
$\alpha=0.5, \lambda=1$ & & \\
$\mathrm{n}=50$ & $0.4871(-0.0129,0.0812)$ & $1.0167(0.0167,0.2429)$ \\
$\mathrm{n}=100$ & $0.4948(-0.0052,0.0561)$ & $1.0131(0.0131,0.1700)$ \\
$\mathrm{n}=300$ & $0.5002(2 \mathrm{e}-04,0.0323)$ & $1.0028(0.0028,0.0969)$ \\
\hline
\end{tabular}

From the results, we can see that as the sample size $\mathrm{n}$ increasing, bias and sd of the CML estimator both decrease, which shows that CML methods are reliable, especially for large sample sizes, which shows that the CML estimators are convergent. 


\section{APPLICATION}

In this section, we apply the BINAR (1) model with geometric innovation to the offence data in Track 1706 in Pittsburgh. The data are available from the section about Crime data at the Forecasting Principles site (http://www.forecastingprinciples.com). The time series data refer to thief data consisted of 144 monthly observations, starting from January 1990 to December 2001.

The counts of thefts data for the Track 1706 has mean value and variance equal to 7.083017 .6206 , respectively, which shows that the data is overdispersed. Therefore, the BINAR(1) model with geometric innovation may be appropriate for the data. The sample path, ACF, PACF of the time series are plotted in Figures 1-2. The sample path shows that the time series is stationary. Then ACF and PACF imply that the first-order auto-regressive model is appropriate for the time series data. We also apply another two models to fit the time series data. One is a BINAR(1) model with Poisson distribution marginal, denoted by PBINAR (1) model, the other model is a INAR(1) model based on binomial thinning operator, denoted by common INAR(1)model [4]. We calculate the CML estimator, standard error (SE), and the values of AIC. The results are reported in Table 2. The GBINAR(1) model has the smallest AIC. Therefore the GBINAR(1) model is more appropriate for the time series data.

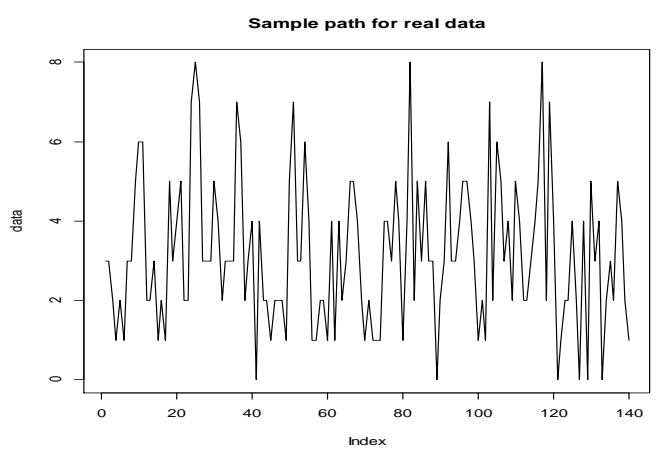

Figure 1: The plot of sample path for real data
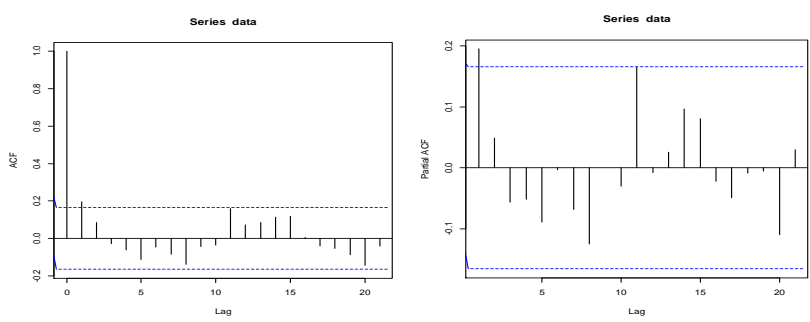

Figure 2: Plots of ACF and PACF for real data

Table 2: Parameter Estimates for the Real Data

\begin{tabular}{|llll|}
\hline CML estimators & $\begin{array}{l}\text { PBINAR(1) } \\
\text { Estimate (SE) }\end{array}$ & $\begin{array}{l}\text { Common INAR(1) } \\
\text { Estimate (SE) }\end{array}$ & $\begin{array}{l}\text { GBINAR(1) } \\
\text { Estimate (SE) }\end{array}$ \\
\hline$\hat{\alpha}$ & $0.2014(0.0032)$ & $0.3201(0.0021)$ & $0.1010(0.0011)$ \\
$\hat{\lambda}$ & $3.4797(0.0407)$ & $2.6693(0.0104)$ & $2.8302(0.0034)$ \\
Log-likelihood & & & \\
AIC & -384.68 & -357.32 & -283.95 \\
\hline
\end{tabular}

\section{CONCLUSIONS}

In this paper, we propose a new stationary INAR(1) model based on binomial thinning operator denoted as BINAR(1) model. The model is constructed by considering the innovation for different dispersed data. The CML method is applied to estimate the para-meters. We considered one specific models, in which the innovation follows geometric distribution, denoted as GBINAR(1)model.The GBINAR(1) model can deal with over-dispersed integer-valued time series data. The performance of CML estimators are evaluated by simulation study, which shows that the CML estimators are reliable, especially for large sample size. A real data example shows that the BINAR(1) model with geometric innovation is appropriate for the thefts data.

There are some possibilities for future research. One can generalize the BINAR(1) model based on random coefficient binomial thinning operator to make the model proposed more flexible with respect to real data applications. Another possible extension is to consider other innovation distributions such as negative binomial distribution and other gamma distributions.

\section{ACKNOWLEDGMENTS}

The authors thank the editors and the two referees for carefully reading the paper and for their valuable suggestions and comments, which greatly improved the paper. The authors also thank the Conference of ICNMIM.

\section{REFERENCES}

[1] Sara, H. 2018. Knowledge grid model in facilitating knowledge sharing among big data community. Acta Informatica Malaysia, 2 (1), 17-18.

[2] Cecilia, A., Rusli, A., Rodziah, A., Yusmadi, Y.J. 2018. Theoretical retical aspect in formulating assessment model of big data analytics environment. Acta Mechanica Malaysia, 1 (1), 16-17.
[3] Steutel, F.W., van Harn, K. 1979. Discrete analogues of selfdecomposability and stability. Applied Probability, 7 (5), 893-899.

[4] Al-Osh, M.A., Alzaid, A.A. 1987. First order integer-valued autoregressive (INAR(1)) process. Jounal of Time series Analysis, 8 (4), 261-275.

[5] Mckenzie, E. 1985. Some simple models for discrete variate time series. Water Resour. Bull, 21 (4), 645-650.

[6] Latour, A. 1990. Existence and stochastic structure of a non-negative integer-valued autoregressive process. Journal of Applied Probability, 27 (5), 314-324.

[7] Weiß, C.H. 2008. Thinning operations for modeling time series of counts-a survey. Advanced Statistics Analysis, 92 (6), 319-341.

[8] Sebastian, S., Weiß, C.H. 2014. Compound Poisson INAR(1) processes: Stochastic properties and testing for overdispersion. Computational Statistics and Data Analysis, 77, 267-284.

[9] Zhu, F. 2012. Zero-inflated Poisson and negative binomial integervalued GARCH models. Journal of Statistical Planning and Inference, 142 (4), 826-839.

[10] Cui, Y., Robert, L. 2010. Inference in binomial AR(1) models. Statistics and Probability Letters, 80, 1985-1990.

[11] Jia, B., Dehui, W., Haixiang, Z. 2013. A study for missing values in PINAR(1) processes. Communications in Statistics: Theory and Methods,doi:10.1080/03610926.2012.717664.

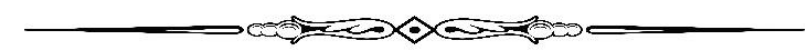

